\title{
Effects of an internal medicine floor interdisciplinary team on hospital and clinical outcomes of seniors with acute medical illness
}

\author{
Ji Won Yoo, ${ }^{1,7}$ Sulgi Kim, ${ }^{3}$ Haesun Seol, ${ }^{4}$ Sun Jung Kim, ${ }^{9}$ Janet Miyoung Yang, ${ }^{2}$ Woo Sang Ryu ${ }^{8}$ \\ Too Jae Min, ${ }^{8}$ Jong Bum Choi, ${ }^{10}$ Minkyung Kwon ${ }^{10}$ and Shunichi Nakagawa, ${ }^{5,6}$
}

\begin{abstract}
${ }^{1}$ Department of Internal Medicine and Institute of Gerontology, University of Michigan Medical School, ${ }^{2}$ Department of Internal Medicine, Saint Joseph Mercy Hospital, Ann Arbor, Michigan, ${ }^{3}$ Department of Epidemiology, School of Public Health, University of Washington, Seattle, Washington, ${ }^{4}$ Federally Qualified Health Center, VNA Health Center, Bensenville, Illinois, ${ }^{5}$ Department of Geriatrics and Palliative Medicine, Mount Sinai School of Medicine, New York, ${ }^{6}$ Geriatric Research, Education and Clinical Center (GRECC), James J. Peters Veterans Affairs Medical Center, Bronx, New York, USA, ${ }^{7}$ Department of Internal Medicine, ${ }^{8}$ Center of Clinical Education, Korea University College of Medicine, ${ }^{9}$ School of Public Health, Yonsei University, and ${ }^{10}$ Center for Clinical Education, Yonsei University College of Medicine, Seoul, Republic of Korea
\end{abstract}

Aim: To examine whether an internal medicine interdisciplinary floor team enhances the hospital and clinical outcomes for seniors with acute medical illness.

Methods: Seniors admitted to medical floor teaching services of a USA teaching hospital were recruited and allocated to the interdisciplinary (ITD; $n=236)$ and usual care teams $(n=248)$. Compared with the usual care team, the interdisciplinary team physicians carried out daily "geriatric" assessment and management, and led the interdisciplinary team meeting designed for improving interprofessional collaboration.

Results: After controlling for patient and physician characteristics, the mean hospital length of stay in the ITD team (6.1 days; $95 \%$ CI 5.2-7.7 days) was 0.7 days shorter than that in the usual care team (6.8 days; $95 \%$ CI 5.7-8.3 days; $P=0.008)$. There was no significant difference in delirium and 30-day hospital readmission between care groups.

Conclusions: Notwithstanding partly positive associations, the results from the present study suggest that interdisciplinary team-based care is, at best, associated with enhancing the clinical and hospital outcomes for seniors with acute medical illness. Geriatr Gerontol Int 2013; 13: 942-948.

Keywords: clinical education, geriatric assessment, hospital medicine, interdisciplinary team, quality improvement.

\section{Introduction}

Older adults account for $40 \%$ of USA hospital admissions. ${ }^{1}$ When observed in parallel with a corresponding increase in life expectancy, older adults' hospital admissions have been on the rise. ${ }^{1}$ Hospitalized seniors are vulnerable to the development of a complicated hospital course and catastrophic consequences, including disability and nursing home entry, even by a bout of severely acute medical illness. ${ }^{2}$

Accepted for publication 11 December 2012.

Correspondence: Dr Ji Won Yoo MD MSc, Department of Internal Medicine and Institute of Gerontology, University of Michigan Medical School, 300 North Ingalls Building, Room 932, Ann Arbor, MI 48109, USA. Email: yoojiw@trinity-health.org
In 2008, the Institute of Medicine noted that unless academic health centers take action immediately, the healthcare workforce will be unable to effectively meet the needs of this growing population. ${ }^{3}$ In response to these urgent needs, several educational initiatives have been suggested for improving medical knowledge and clinical skills of resident physicians. ${ }^{4-8}$ However, little is known about the actual hospital and clinical outcomes of hospitalized seniors since introducing educational initiatives for resident physicians.

A serial of studies have linked specific collaborative behaviors to improved health outcomes for hospitalized seniors. ${ }^{9-11}$ These studies show that practices as specific as providing information when it is not explicitly asked for and facilitating more frequent communication opportunities among healthcare providers have been identified as improving interprofessional collaboration. 
Innovations to incorporate an interprofessional collaboration into internal medicine teaching services are urgently required to improve quality of care for hospitalized seniors. To meet this need, since 2007, the Cleveland Clinic Health System in the USA and Korea University in Korea have been collaborating to develop the educational programs for graduate medical trainees, and to evaluate these programs. ${ }^{12}$ This collaboration primarily puts an emphasis on improving the quality of care of hospitalized older adults. ${ }^{12}$ As a result, an interdisciplinary internal medicine teaching service model is developed to enhance: (i) geriatric assessment and management; and (ii) interprofessional collaboration among healthcare professionals.

The present study aimed to evaluate the clinical and hospital outcomes of seniors with acute medical illness cared for by an interdisciplinary team in an internal medicine floor teaching service of a USA academic medical center.

\section{Methods}

The study site was a USA metropolitan hospital affiliated with an academic center (485 certified beds). This Graduate Medical Education program is an Accreditation Council for Graduate Medical Education (ACGME) accredited internal medicine program. All physicians participating to the study complete $6 \mathrm{~h}$ of American Medical Association (AMA) physician's recognition awards (PRA) category 1 by either onsite (noon conference) or online continuous medical education (CME) before the patient enrolment. The title and topics of CME are "Enhancing Quality of Care for Hospitalized Seniors", and (i) "Physical Function Assessment"; (ii) "Hospital-associated Frailty and Disability"; (iii) "Cognitive Function Assessment (Mini-Mental Status Examination [MMSE])", (iv) "Delirium Diagnosis (Confusion Assessment Method [CAM]), Prevention and Management"; (v) "Medication Reconciliation During and After Hospital Care"; and (vi) "Interdisciplinary Team Care for Hospitalized Seniors". ${ }^{12-14}$ The present study was approved by the Institutional Review Board of the Cleveland Clinic Health System. Informed consents are obtained from both participating physicians and patients or their informal primary caregivers when patients could not make their own decisions.

\section{Patient inclusion and exclusion criteria}

The patient enrolment was between January and April 2010 (16 weeks). Patients were chosen based on the following inclusion criteria: (i) admission to medical floors or telemetry units under teaching service; (ii) age 65 years or older; and (iii) community-dwelling person before hospital admission. Exclusion criteria were: (i) hospice enrolee; (ii) admission to intensive care unit; and (iii) admission to non-teaching medicine floor services. A total of 598 patients met the initial eligibility. Figure 1 shows the flow chart of patient enrolment, allocation, follow-up and analysis according to CONsolidated Standards of Reporting Trials (CONSORT).

\section{Sample size determination}

We estimated that a sample size of 234 was required for each study group based on the expected mean hospital length of stay of the usual care (7 days) and interdisciplinary (6 days) teams (expected standard deviation: 3 days) with an alpha of $5 \%$ and a power of $95 \% .{ }^{15}$

\section{Study protocol: Interdisciplinary care team of geriatric assessment and management}

As opposed to their counterparts in the usual care team, the physicians in the interdisciplinary (ITD) team were required to complete daily "geriatric" assessment and management from hospital admission to discharge as follows: (i) physical function assessment using activities of daily living (ADL); (ii) cognitive assessment (MMSE), (iii) delirium assessment (CAM); (iv) medication reconciliations; and (v) the minimization of sleep disturbance (i.e. sleep protocol) or physical restraints unless medically necessary. The compliance of study protocol was determined whether a physician completed daily assessment and management $80 \%$ or more: For example, if a total number of documented items was 34 of 40 items (hospital length of stay is 8 days, $8 \times 5$ items $=40$ items), we considered this patient care as compliant to study protocol. However, if a total number of documented items was just 30 of 40 items, we considered this patient care as non-compliant to study protocol and excluded this patient from the analysis. There were 52 occasions of patient drop-out because of poor compliance to study protocol in the interdisciplinary team.

\section{Study protocol: ITD team meeting}

The ITD team meeting consisted of physicians, nurses, pharmacists, social workers, nutritionists, and physical/ occupational/speech and language therapists. The ITD team physicians attended and led the ITD team meeting to enhance interprofessional collaboration among healthcare providers. The average ITD team meeting time was $45 \mathrm{~min}$. The frequency of ITD team meetings was three times per week. The place of this meeting was the medicine floor conference room. Selected patients were discussed during the ITD team meeting. The usual care team physicians voluntarily attended and led the ITD team meeting on 16 occasions. These occasions were considered as contamination, and these were withdrawn from the analysis. 


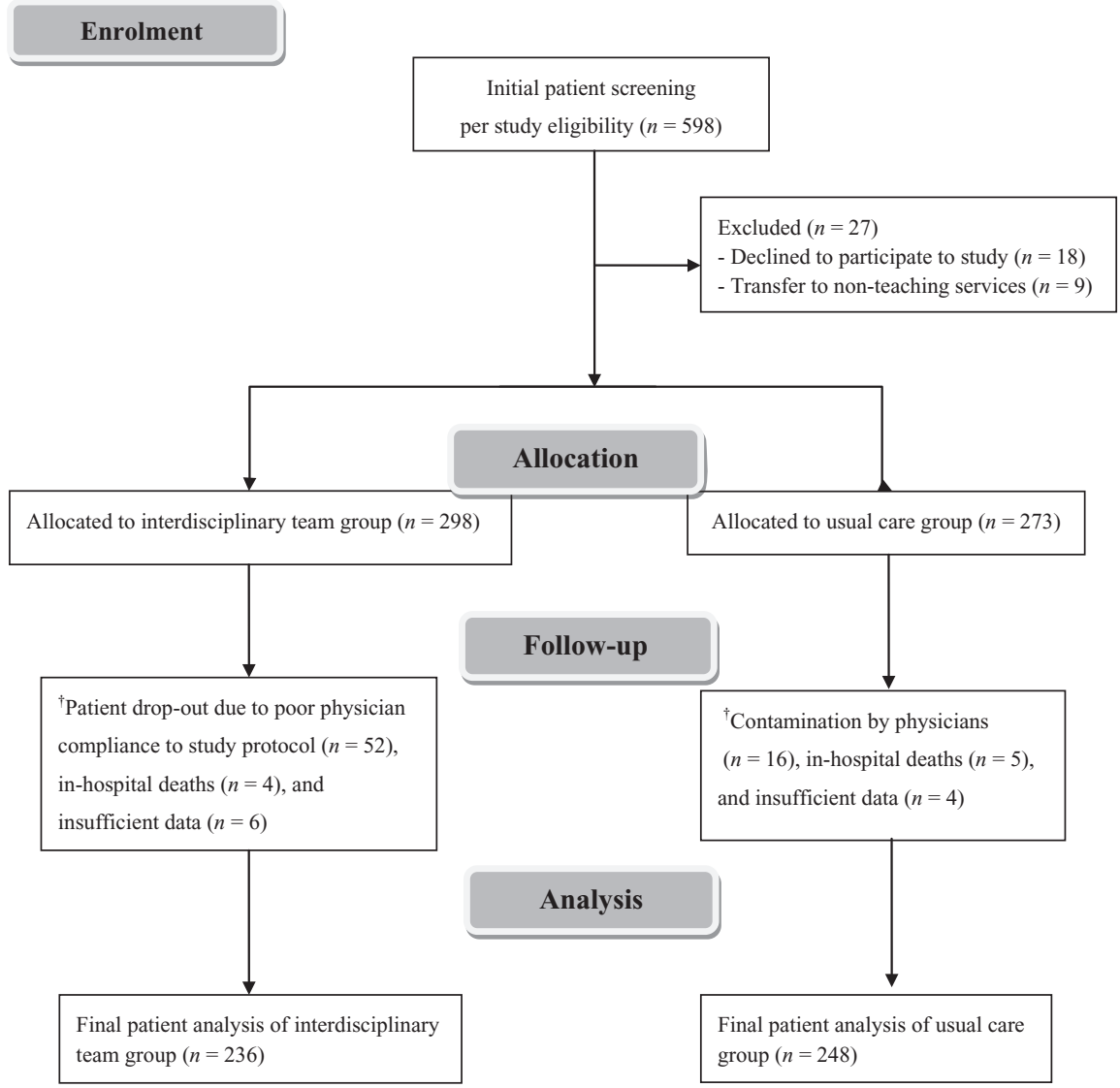

Figure 1 Flow chart of patient enrolment, allocation, follow up and analysis. ${ }^{\mathrm{t}}$ Given the number of drop-out cases, intention to treat analysis was carried out. The results did not change after intention to treat analysis.

\section{Patient and patient and physician allocation}

The study coordinator, who did not participate in patient care, allocated patients by matching demographics, severity of illness and admission diagnoses. The study coordinator matched physician category and years of experience between care groups. Physicians were aware of their team allocations. A total of 11 physicians (4 attending and 7 training physicians) allocated to the ITD team and eight physicians ( 3 attending and 5 training physicians) allocated to the usual care team declined to participate in the study. All participating training physicians adhered to the ACGME-defined limits on duty hours and admissions.

\section{Main outcomes}

(1) Clinical outcomes: Delirium

The CAM was used by participating physicians to detect delirium. Researchers reviewed physicians' daily progress notes as to whether delirium occurred. The validity of CAM for hospitalized older adults has been discussed elsewhere. ${ }^{16}$

(2) Hospital outcomes: Hospital length of stay and 30-day hospital readmission

Researchers were able to capture hospital length of stay and 30-day hospital readmission data through hospital administrative data.

\section{Participant characteristics}

\section{Patient characteristics}

Participating patient characteristics were age, sex, ethnicity, education, marital status, severity of illness, physical function, cognition, home or day services and admission diagnoses. Demographics (age, sex, ethnicity, education and marital status data) were collected from the hospital administrative database. The All Patient Refined Diagnostic Related Group (APR-DRG) severity of illness classification system was used to estimate the severity of illness. The APR-DRG data were gathered from the $3 \mathrm{M}$ Health Information System (Wallingford, CT, USA). The APR-DRG data were collected from the Department of Medical Operations, which was unaware of the study objectives and was abstracted by the researchers. The validity of APR-DRG severity of illness in inpatient geriatric care has been discussed elsewhere. ${ }^{17}$ Physical functions were assessed by the same nursing staff using the Katz Index of independence of ADL. The Katz Index ranged from 0 (most dependent) to 6 (most independent). The severity of physical impairment was categorized by the Katz Index: none (6), mild ( 4 to 5 ) and moderate to severe (0 to 3$)$. The Katz Index has been shown to have acceptable internal consistency $(\alpha=0.87$ ) and validity ( $\kappa=0.74$ to 0.88 ) when diverse health professionals carry out the assessment in 
a hospital setting. ${ }^{18,19}$ The interobserver agreement $(\kappa=0.64)$ and intra-observer reproducibility $(\kappa=0.88)$ of the Katz Index of the present study were relatively high. Cognitive impairment and home or day care services data were collected from clinical and administrative data. Admitting diagnoses at hospital admission were grouped into the following eight groups using the International Classification of Diseases, Ninth Revision, Clinical Modification (ICD-9-CM) codes: (i) cardiovascular; (ii) respiratory; (iii) cancer and blood organ; (iv) infectious; (v) endocrine and metabolic; (vi) digestive and urogenital tract; (viii) neurological; and (viii) other diseases.

\section{Physician characteristics}

As for attending physicians, each team consisted of internal medicine board-certified physicians. None of physicians had additional board qualifications on geriatric medicine. A total of 54 (27 attending and 27 training) physicians in the ITD team and 53 (24 attending and 29 training) physicians in the usual care team participated in the study.

\section{Statistical analysis}

Bivariate comparisons of patient and physician characteristics between care teams were examined using $\chi^{2}$-tests to compare categorical data, and $t$-tests to compare continuous data. All reported $P$-values are two-sided throughout, and $P<0.05$ is considered statistically significant. We estimated multivariate logistic regressions of "30-day hospital readmission" and "delirium" controlling for patient and physician characteristics to compute odds ratios (OR) along with corresponding $95 \%$ confidence intervals (CI). ${ }^{20}$ Odds ratio $>1$ indicates that the probability in the ITD team is higher than that in the usual care team. We used generalized linear models (GLM) with a log link function and gamma distribution to fit skewed hospital length of stay. ${ }^{21}$ The predicted hospital length of stay difference (usual care minus ITD teams) with the corresponding 95\% CI was derived from general linear models. All data procedures and analyses were carried out using SAS statistical software version 9.2 (SAS Institute, Cary, NC, USA).

\section{Results}

\section{Patient and physician characteristics}

A total of 248 patients were cared for by the usual care team and 236 patients were cared for by the ITD team. The patient and physician characteristics between care teams were not statistically different. Tables 1 and 2 present the patient and physician characteristics by care teams.

\section{Clinical outcomes: delirium}

Table 3 presents the clinical outcomes by care teams. There was no significant difference in mean probabilities of delirium between care teams.

\section{Hospital outcomes: Hospital length of stay and 30-day hospital readmission}

Table 4 presents the hospital outcomes by care teams. The mean hospital length of stay in the ITD team (6.1 days; 95\% CI 4.2-8.7 days) was 0.7 days shorter than that in the usual care team ( 6.8 days; $95 \%$ CI $4.7-9.3$ days; $P=0.008)$. There was no significant difference in mean probabilities of 30-day hospital readmission between care teams.

\section{Assessment of variance, sensitivity, model fit and intention to treat}

The total variances of hospital length of stay explained by regression were $38 \%$. The present results were consistent across numerous alternative specifications including an ordinary least squares regression. All logistic regressions fit well as determined by HosmerLemeshow test results $(P=0.67,30$-day hospital readmission; $P=0.35$, delirium). There were 62 dropout cases in the ITD team: 52 cases of poor physician compliance to study protocol; four cases of in-hospital deaths; and six cases of insufficient data. There were 25 drop-out cases in the usual care team: 16 cases of contamination by physicians; five cases of in-hospital deaths; and four cases of insufficient data. Given a certain number of drop-out cases, intention to treat (ITT) analysis is carried out. The results were not changed even after ITT analysis.

\section{Discussion}

To the best of our knowledge, the present study is the first study investigating the "clinical and hospital outcomes" of hospitalized seniors cared for by an internal medicine interdisciplinary team. There are notable reductions in days of hospital length of stay when participating patients were cared for by the interdisciplinary team. However, reductions in delirium and 30-day hospital readmission in the patients of the interdisciplinary team did not occur.

The current practice style, leaving the hospital "quicker-sicker" trend related to the introduction of the diagnosis-related group (DRG) might have contributed to greater instability at hospital discharge..$^{22,23}$ The consequences of this "quicker-sicker" trend have been linked to poor discharge outcomes (e.g. more frequent transitions to a residential facility and more frequent, earlier hospital readmissions) compared with similar 
Table 1 Patient characteristics

\begin{tabular}{|c|c|c|c|c|}
\hline \multicolumn{2}{|l|}{ Characteristics, \% (n) } & \multirow{2}{*}{$\begin{array}{l}\text { Usual care team } \\
(n=248)\end{array}$} & \multirow{2}{*}{$\begin{array}{l}\text { ITD team } \\
(n=236)\end{array}$} & \multirow{2}{*}{$\begin{array}{l}P \\
0.51\end{array}$} \\
\hline Age & 80 years or older & & & \\
\hline Female & & $59(146)$ & $58(138)$ & 0.49 \\
\hline \multirow[t]{3}{*}{ Ethnicity } & African Americans & $53(132)$ & $51(121)$ & 0.62 \\
\hline & Caucasians & $36(89)$ & $37(87)$ & \\
\hline & Others & $11(27)$ & $12(28)$ & \\
\hline \multicolumn{2}{|c|}{ High school graduate or higher education } & $43(107)$ & $41(97)$ & 0.40 \\
\hline \multicolumn{2}{|c|}{ Never married, widowed or divorced } & $32(79)$ & $35(83)$ & 0.68 \\
\hline \multirow[t]{4}{*}{ APR-DRG severity of illness } & Mild & $9(23)$ & $10(24)$ & 0.23 \\
\hline & Moderate & $33(81)$ & $36(85)$ & \\
\hline & Major & $42(104)$ & $40(94)$ & \\
\hline & Extreme & $16(40)$ & $14(33)$ & \\
\hline \multirow[t]{3}{*}{ Physical impairment at admission } & None & $38(94)$ & $35(82)$ & 0.28 \\
\hline & Mild & $45(111)$ & $43(102)$ & \\
\hline & Moderate to severe & $17(43)$ & $22(52)$ & \\
\hline Cognitive impairment & & $18(45)$ & $22(52)$ & 0.16 \\
\hline Home or day care services & & $19(47)$ & $21(49)$ & 0.37 \\
\hline \multirow[t]{8}{*}{ Admission diagnoses } & Cardiovascular diseases & $13(32)$ & $15(35)$ & 0.35 \\
\hline & Respiratory diseases & $17(42)$ & $14(33)$ & \\
\hline & Cancer and blood organ diseases & $8(20)$ & $8(19)$ & \\
\hline & Infectious diseases & $16(39)$ & $17(40)$ & \\
\hline & Endocrine/metabolic diseases & $8(20)$ & $9(21)$ & \\
\hline & Digestive/urogenital tract diseases & $19(47)$ & $18(43)$ & \\
\hline & Neurological diseases & $10(25)$ & $11(26)$ & \\
\hline & Others & $9(23)$ & $8(19)$ & \\
\hline
\end{tabular}

APR-DRG, all patient refined-diagnosis related group; ITD, interdisciplinary.

Table 2 Physician characteristics

\begin{tabular}{llllr}
\hline$n(\%)$ or mean (standard deviation) & $\begin{array}{l}\text { Physicians at usual } \\
\text { care team }(n=54)\end{array}$ & $\begin{array}{l}\text { Physicians at } \\
\text { interdisciplinary } \\
\text { care team }(n=53)\end{array}$ & $P$ \\
\hline Attending physician & Hospitalist & 13 & 11 & 0.20 \\
& Non-hospitalist & 14 & 13 & 0.19 \\
Training physician & Years in experience & $8.5(6.1)$ & $8.0(5.7)$ & 0.43 \\
& PGY-1 & 11 & 9 & \\
& PGY-2 & 8 & 8 & \\
& PGY-3 or higher & 8 & &
\end{tabular}

PGY, postgraduate year.

outcomes before the DRG introduction..$^{22,23}$ Observational studies have found that the trend of a shortened hospital stay was associated with an increased risk of early hospital readmission rate. ${ }^{24,25}$ The present findings might replicate the absence of an inverse relationship of the previous intervention studies. ${ }^{11,12,26}$ An interdisciplinary team might have played a "buffering role" of an inverse relationship between shortened hospital length of stay and early hospital readmission.

Hospital length of stay has been considered as the indicator of evaluating efficiency of hospital care. ${ }^{23,24}$
The reduction in hospital length of stay in the ITD team holds substantial implications as a tool for potentially improving hospital reimbursements by shortening hospital stay and increasing the Agency for Healthcare Research and Quality (AHRQ) quality index composite. ${ }^{31}$ Starting in 2013, $\%$ of hospitals' reimbursements will be increased when a hospital meets the AHRQ's composite score (also knows as value-based incentive payments). ${ }^{1,27}$

We could not find a significant difference in delirium between the care groups. Delirium has been known to 
Table 3 Multivariate adjusted logistic regressions of "delirium"

\begin{tabular}{llllr}
\hline $\begin{array}{l}\text { Mean probability of } \\
\text { delirium, 95\% CI }(\%)\end{array}$ & $\begin{array}{l}\text { Usual care team } \\
(n=248)\end{array}$ & $\begin{array}{l}\text { ITD team } \\
(n=236)\end{array}$ & $\begin{array}{l}\text { †Odds ratio } \\
(95 \% \mathrm{CI})\end{array}$ & $P$ \\
\hline Delirium & $21(16-25)$ & $23(17-28)$ & $1.34(0.73-1.96)$ & 0.34 \\
\hline
\end{tabular}

${ }^{\ddagger}$ The mean probability of delirium with the corresponding $95 \%$ confidence intervals (CI) is derived from multivariate logistic regressions controlling patient and physician covariates. Odds ratio $>1$ indicates that the probability in the interdisciplinary (ITD) team was higher than that in the usual care team.

Table 4 Multivariate adjusted regressions of Hospital Outcomes: "Hospital Length of Stay and 30-day readmission"

\begin{tabular}{lllcc}
\hline $\begin{array}{l}\text { Mean hospital length of stay, } \\
95 \% \text { CI (days) }\end{array}$ & $\begin{array}{l}\text { Usual care team } \\
(n=248)\end{array}$ & $\begin{array}{l}\text { ITD team } \\
(n=236)\end{array}$ & $\begin{array}{l}\text { †Difference, } \\
95 \% \text { CI (days) }\end{array}$ & $P$ \\
\hline Hospital length of stay & $6.8(4.7-9.3)$ & $6.1(4.2-8.7)$ & $0.7(0.1-1.3)$ & 0.008 \\
\hline $\begin{array}{l}\text { Mean probability of 30-day hospital } \\
\text { readmission, 95\% CI (\%) }\end{array}$ & $\begin{array}{l}\text { Usual care team } \\
(n=248)\end{array}$ & $\begin{array}{l}\text { ITD team } \\
(n=236)\end{array}$ & $\begin{array}{l}\text { †Odds ratio } \\
(95 \% \text { CI })\end{array}$ & $P$ \\
\hline 30-day hospital readmission & $16(12-21)$ & $14(9-17)$ & $0.75(0.42-1.15)$ & 0.20 \\
\hline
\end{tabular}

TThe average predicted hospital length of stay difference (usual care minus interdisciplinary [ITD] teams) with the corresponding $95 \%$ confidence intervals (CI) from general linear models of hospital length of stay controlling patient and physician covariates. Odds ratio $>1$ indicated that the probability in the ITD team was higher than that in the usual care team.

be one of the contributors to prolonged hospital stay, especially in older adults. ${ }^{10,13}$ The ineffectiveness of the ITD team might be explained as follows: first, the usual care group physicians were also reminded of preventing and recognizing delirium by didactic education sessions before enrolment. Therefore, gaps in prioritizing the importance of delirium care between these care groups might have been diminished. Second, as had been seen in other intervention studies for hospitalized older adults, it is possible that delirium is underdiagnosed by usual care group physicians. ${ }^{28,29}$ Previous investigations explicate the under-recognition of delirium in usual care groups as follows: difficulty in identifying the hypoactive form of delirium because of such patients' tendency to cooperate with their care; and healthcare professionals' tendency to overlook cognitive assessment in a substantial proportion of older hospitalized patients. ${ }^{28-30}$ These two explanations of ineffectiveness of ITD intervention to prevent the occurrence of delirium appear to be contradictory. However, we cannot discern which explanation would be more convincing than the other. A recent comprehensive review on preventing delirium in older adults showed that the largest effects of interventions were seen in populations with an incidence of delirium above $30 \% .{ }^{30}$ In the present study, the beneficial effects of interventions appeared to diminish at a relatively lower incidence (between $20 \%$ and $25 \%$ ) of delirium.

We acknowledge several limitations in data collection and study design. Because the data collection was limited to a USA institution, a major limitation is lack of generalizability. The study coordinator allocated patients and physicians into care groups. Therefore, selected bias might have occurred during group allocation process. Considering the secondary analysis of clinical data (i.e. cognition), this data collection was not designed for the study, suggesting potential observer variation. There is still a need for further exploration of how the interdisciplinary team enhances clinical and hospital outcomes. Therefore, the findings should be interpreted with caution and considered preliminary until they are confirmed in future studies with more representative data.

\section{Acknowledgments}

This study was funded by a Junior Researcher Seed Grant (American Geriatrics Society) and an Excellence for Graduate Medical Education Grant (Ministry of Education, Science and Technology, Republic of Korea).

\section{Disclosure statement}

All authors report no conflict of interest.

\section{References}

1 Medicare Payment Advisory Commission. Report to the Congress: Medicare Health Care Delivery System. 2012. [Cited 10 Dec 2012] Available from URL: http://www. medpac.gov/documents/cfm 
2 Ferrucci L, Guralnik JM, Pahor M, Corti MC, Havik RJ. Hospital diagnoses, Medicare charges, and nursing home admissions in the year when older persons become severely disabled. JAMA 1997; 277: 728-734.

3 Institute of Medicine. Committee on the Future Health Care Workforce for Older Americans. Retooling for An Aging America: Building the Health Care Workforce. Washington, DC: National Academies Press, 2008.

4 Fernandez HM, Callahan KE, Likourezos A, Leipzig RM. House staff member awareness of older inpatients' risks for hazards of hospitalization. Arch Intern Med 2008; 168: 390-396.

5 Leipzig RM, Granville L, Simpson D, Anderson MB, Sauvigné K, Soriano RP. Keeping granny safe on July 1: a consensus on minimum geriatrics competencies for graduating medical students. Acad Med 2009; 84: 604610.

6 Westmoreland GR, Counsell SR, Tu W, Wu J, Litzelman $\mathrm{DK}$. Web-based training in geriatrics for medical residents: a randomized controlled trial using standardized patients to assess outcomes. J Am Geriatr Soc 2010; 58: $1163-$ 1169.

7 Moran WP, Zapka J, Iverson PJ et al. Aging Q3: an initiative to improve internal medicine residents' geriatrics knowledge, skills, and clinical performance. Acad Med 2012; 87: 635-642.

8 Fulmer T, Hyer K, Flaherty E et al. Geriatric interdisciplinary team training program: evaluation results. J Aging Health 2005; 17: 443-470.

9 Weller JM, Barrow M, Gasquoine S. Interprofessional collaboration among junior doctors and nurses in the hospital setting. Med Educ 2011; 45: 478-487.

10 Malone ML, Vollbrecht M, Stephenson J, Burke L, Pagel $\mathrm{P}$, Goodwin JS. Acute Care for Elders (ACE) tracker and e-Geriatrician: methods to disseminate ACE concepts to hospitals with no geriatricians on staff. J Am Geriatr Soc 2010; 58: 161-167.

11 Naylor MD, Bowles KH, McCauley KM et al. High-value transitional care: translation of research into practice. J Eval Clin Pract 2011; doi: 10.1111/j.1365-2753.2011.01659.x [Epub ahead of print].

12 Yoo JW, Nakagawa S, Kim S. Delirium and transition to a nursing home of hospitalized older adults: a controlled trial of assessing the interdisciplinary team-based "geriatric" care and care coordination by non-geriatrics specialist physicians. Geriatr Gerontol Int 2012; doi: 10.1111/j.14470594.2012.00905.x [Epub ahead of print].

13 Lyons WL, Landefeld CS. Hospital care. In: Pacala JT, Sullivan GM, eds. Geriatrics Review Syllabus: A Core Curriculum in Geriatric Medicine, 7th edn. New York, NY: The American Geriatrics Society, 2010.

14 Flaherty E, Hyer K, Fulmer T. Team care. In: Halter JB, Ouslander JG, Tinetti ME, Studenski S, High KP, Asthana $\mathrm{S}$, eds. Hazzard's Geriatric Medicine and Gerontology, 6th edn. New York, NY: McGraw-Hill Medical, 2009; Chapter 26.

15 Stokes ME, Davis CS, Koch GG. Categorical Data Analysis Using the SAS System. 2nd ed. Cary, NC: SAS, 2009.
16 Inouye SK, van Dyck CH, Alessi CA, Balkin S, Siegal AP, Horwitz RI. Clarifying confusion: the Confusion Assessment Method: a new method for detection of delirium. Ann Intern Med 1990; 113: 941-948.

17 Pilotto A, Scarcelli C, D'Ambrosio LP et al. All patient refined diagnosis related groups: a new administrative tool for identifying elderly patients at risk of high resource consumption. J Am Geriatr Soc 2005; 53: 167-168.

18 Brorsson B, Asberg KH. Katz index of independence in ADL. Reliability and validity in short-term care. Scand $J$ Rehabil Med 1984; 16: 125-132.

19 Ciesla JR, Shi L, Stoskopf CH, Samuels ME. Reliability of Katz's Activities of Daily Living Scale when used in telephone interviews. Eval Health Prof 1993; 16: 190-203.

20 Hosmer DW, Lemeshow S. Applied Logistic Regression, 2nd edn. Hoboken, NJ: John Wiley \& Sons, Inc, 2005.

21 Manning WG, Basu A, Mullahy J. Generalized modeling approaches to risk adjustment of skewed outcomes data. $J$ Health Econ 2005; 24: 465-488.

22 Kosecoff J, Kahn KL, Rogers WH et al. Prospective payment system and impairment at discharge: the "quicker and sicker" story revisited. JAMA 1990; 264: 1980-1983.

23 Grabowski DC, Afendulis CC, McGuire TG. Medicare prospective payment and the volume and intensity of skilled nursing facility services. J Health Econ 2011; 30: 675-684.

24 Bueno H, Ross JS, Wang Y et al. Trends in length of stay and short-term outcomes among Medicare patients hospitalized for heart failure, 1993-2006. JAMA 2010; 303: 2141-2147.

25 Baker DW, Einstadter D, Husak SS, Cebul RD. Trends in postdischarge mortality and readmissions: has length of stay declined too far? Arch Intern Med 2004; 164: 538-544.

26 McMahon GT, Katz JT, Thorndike ME, Levy BD, Loscalzo J. Evaluation of a redesign initiative in an internalmedicine residency. $N$ Engl J Med 2010; 362: 1304-1311.

27 Medicare Payment Advisory Commission (MedPAC). Hospital inpatient and outpatient services: assessing payment adequacy and updating payments. MedPAC report to Congress. Washington DC: Department of Health and Human Services, 2011, pp. 38-66.

28 Inouye SK, Leo-Summers L, Zhang Y, Bogardus ST Jr, Leslie DL, Agostini JV. A chart-based method for identification of delirium: validation compared with interviewer ratings using the confusion assessment method. $J \mathrm{Am}$ Geriatr Soc 2005; 53: 312-318.

29 Steis MR, Fick DM. Delirium superimposed on dementia: accuracy of nurse documentation. J Gerontol Nurs 2012; 38: 32-42.

30 Hempenius L, van Leeuwen BL, van Asselt DZ et al. Structured analyses of interventions to prevent delirium. Int $J$ Geriatr Psychiatry 2011; 26: 441-450.

31 Medicare Payment Advisory Commission. Medicare Payment Policy: Assessing payment adequacy and updating payments in fee-for-service Medicare. Medicare Payment Advisory Commission report to Congress 2012: 31-44 [Cited 10 Dec 2012.] Available from URL: http://www.medpac.gov/ documents/cfm 